\title{
Molecular detection and genotyping of Enterocytozoon bieneusi in pet dogs in Xinjiang, Northwestern China
}

Yangwenna Cao, Qinglin Tong, Chenhao Zhao, Aikebaierjiang Maimaiti, Liwen Chuai, Junjie Wang, Dingyun Ma, and Meng Qi*

College of Animal Science, Tarim University, Alar, Xinjiang 843300, PR China

Received 4 January 2021, Accepted 4 July 2021, Published online 20 July 2021

\begin{abstract}
Enterocytozoon bieneusi is an obligate intracellular parasitic fungi that infects a wide range of mammalian hosts. However, the literature is lacking information regarding the presence and diversity of E. bieneusi genotypes in domesticated dogs in Northwestern China. Fecal samples from 604 pet dogs were obtained in 5 cities (Urumqi, Korla, Hotan, Aksu, and Shihezi) in Xinjiang. Screening for E. bieneusi was performed, and isolates were genotyped via nested-PCR amplification of the internal transcribed spacer (ITS) of nuclear ribosomal DNA. The infection rate of E. bieneusi was $6.3 \%$ (38/604). The prevalence of E. bieneusi infections in adult animals ( $>1$ year, 10.3\%, 15/145) was higher than that in younger $(\leq 1$ year) dogs $(5.0 \%, 23 / 459)$, which was statistically significant $(p=0.021)$. No significant difference was observed between the different collection sites or between sexes. Eight distinct genotypes were identified, including 5 known genotypes (PtEb IX, EbpC, D, CD9, and Type IV) and 3 novel genotypes (CD11, CD12, CD13). The most prevalent was genotype PtEb IX, being observed in 50.0\% (19/38) of the samples, followed by $\operatorname{EbpC}(31.6 \%, 12 / 38), \mathrm{D}(5.3 \%, 2 / 38)$, and the remaining genotypes (CD9, Type IV, CD11, CD12, and CD13) were observed in 1 sample $(2.6 \%, 1 / 38)$ each. These findings suggest that genotypes PtEb IX and CD9 are canine hostadapted, and likely pose little risk of zoonotic transmission. Moreover, known zoonotic genotypes EbpC, D, and Type IV represent a public health concern and should undergo further molecular epidemiological investigation.
\end{abstract}

Key words: Enterocytozoon bieneusi, Pet dogs, Genotype, Infection rate.

Résumé - Détection moléculaire et génotypage d'Enterocytozoon bieneusi chez les chiens de compagnie au Xinjiang, nord-ouest de la Chine. Enterocytozoon bieneusi est un champignon intracellulaire, parasite obligatoire, qui infecte un large éventail d'hôtes mammifères. Cependant, la littérature manque d'informations concernant la présence et la diversité des génotypes d'E. bieneusi chez les chiens domestiques dans le nord-ouest de la Chine. Des échantillons fécaux de 604 chiens de compagnie ont été obtenus dans 5 villes (Urumqi, Korla, Hotan, Aksu et Shihezi) au Xinjiang. Le dépistage d'E. bieneusi a été effectué et les isolats ont été génotypés par amplification PCR nichée de l'espaceur transcrit interne (ITS) de l'ADN ribosomique nucléaire. Le taux d'infection d'E. bieneusi était de $6,3 \%$ (38/604). La prévalence des infections à $E$. bieneusi chez l'adulte $(>1$ an, 10,3\%, 15/145) était plus élevée que chez les chiens plus jeunes $(\leq 1$ an) $(5,0 \%, 23 / 459)$, ce qui était statistiquement significatif $(p=0,021)$. Aucune différence significative n'a été observée entre les différents sites de collecte ou entre les sexes. Huit génotypes distincts ont été identifiés, dont 5 connus (PtEb IX, EbpC, D, CD9, Type IV) et 3 nouveaux (CD11, CD12, CD13). Le plus répandu était le génotype PtEb IX, observé dans 50,0 \% (19/38) des échantillons, suivi par EbpC $(31,6 \%, 12 / 38), \mathrm{D}(5,3 \%, 2 / 38)$ et les génotypes restants (CD9, Type IV, CD11, CD12 et CD13) ont été observés dans 1 échantillon $(2,6 \%, 1 / 38)$ chacun. Ces résultats suggèrent que les génotypes PtEb IX et CD9 sont adaptés à l'hôte canin et présentent probablement un faible risque de transmission zoonotique. De plus, les génotypes zoonotiques connus EbpC, D et Type IV représentent un problème de santé publique et nécessitent une enquête épidémiologique moléculaire plus approfondie.

\section{Introduction}

Microsporidia are diverse and ubiquitous obligate intracellular parasitic fungi, with diverse hosts ranging from protists to vertebrates, up to and including humans [16, 27]. There are nearly 1500 described microsporidian species in over

*Corresponding author: qimengdz@163. com
200 genera [13]. Of the 17 microsporidian species known to be human pathogens, Enterocytozoon bieneusi is the most prevalent one that infects the gastrointestinal tract and is responsible for $90 \%$ of human microsporidiosis cases reported globally [16].

First detected in 1985, E. bieneusi was isolated from a Haitian AIDS patient suffering from severe diarrhea [8]. As 
an emerging infectious agent, E. bieneusi infection is characterized by acute or chronic diarrhea, malabsorption, and/or wasting $[17,26]$. Additionally, immunocompetent individuals with asymptomatic infections are also commonly reported worldwide [23, 27]. Furthermore, E. bieneusi has also been detected in animals. Some zoonotic genotypes are commonly identified both in humans and synanthropic animals, which suggests a potential for zoonotic transmission [13].

Due to the small size of its spores and the uncharacteristic staining properties of this organism, it is difficult to detect E. bieneusi by routine microscopy. As such, PCR is currently the most reliable tool for diagnosis of E. bieneusi infections [13]. Currently, amplification and sequencing of the ribosomal internal transcribed spacer (ITS) is widely used to identify and genotype E. bieneusi strains [13]. To date, over 500 E. bieneusi genotypes have been defined, which constitute 11 phylogenetic groups with distinct differences in their host specificity and zoonotic potential [11-13, 16]. Group 1 and Group 2 include most of the potentially zoonotic genotypes, whereas the remaining clusters exhibit strong host specificity [16].

The rate of pet ownership is increasing globally, as animals enrich the lives of humans. In China, it has been estimated that approximately $17 \%$ of households own companion animals, dogs being the most common numbering 62 million in 2019 (https://www.chyxx.com/industry/202006/874331.html). Although there are benefits of animal companionship, pet animals can carry diseases that may be transmitted to humans, leading to a potential threat to public health. Reports have documented the prevalence and genotype distributions of E. bieneusi in dogs worldwide, and the prevalence ranges from $0.8 \%$ to $25.8 \%$ (Table 2). In China, there are also some reports of the occurrence of E. bieneusi in dogs is the east of the country [15, 28, 30, 31]. However, data from Northwestern China are lacking. Therefore, this study aimed to investigate the occurrence and genetic diversity of E. bieneusi in pet dogs in the Xinjiang Uygur Autonomous Region (hereinafter referred to as Xinjiang), Northwest China, and to assess the zoonotic potential of any detected E. bieneusi strains.

\section{Materials and methods}

\section{Ethics statement}

This study was conducted in accordance with the Chinese Laboratory Animal Administration Act (1988). The Ethics Committee of Tarim University (protocol number: DW201802003) reviewed and approved this sampling protocol. Appropriate permissions and assistance were obtained from the directors or the owners at each pet hospital, shop, or kennel, before collecting fecal samples from the dogs.

\section{Sample collection and DNA extraction}

A total of 604 individual fecal samples were obtained from dogs in 5 cities (Urumqi, Korla, Hotan, Aksu, and Shihezi) in Xinjiang, China, between April 2018 and June 2020. Within the collection sites, 3 types of sample sources were included: 8 pet hospitals, 17 pet shops, and 6 kennels. The age of sampled pet dogs ranged from 2 months to 13 years.
The samples were collected directly from the rectum of each animal or immediately after defecation and picked up using sterile disposable gloves. Identification number, collection site, sample source, sex, and age were recorded with the help of the site directors or dog owners. The stools were non-diarrheal at the time of sampling. All samples were placed on ice in separate containers and transported to the laboratory immediately. Prior to DNA extraction, fecal samples were stored at $4{ }^{\circ} \mathrm{C}$.

\section{DNA extraction}

Total genomic DNA was extracted from each fecal sample using an E.Z.N.A. ${ }^{\circledR}$ Stool DNA kit (D4015-02, Omega Biotek Inc., Norcross, GA, USA). Briefly, about $200 \mathrm{mg}$ of each fecal sample were placed in a $2 \mathrm{~mL}$ centrifuge tube containing $200 \mathrm{mg}$ of glass beads and placed on ice. Next, $300 \mu \mathrm{L}$ of buffer SP1 and proteinase $\mathrm{K}$ were added, and the tubes were incubated at $70{ }^{\circ} \mathrm{C}$ for $10 \mathrm{~min}$. Subsequently, all the procedures outlined in product manual were performed according to the kit manufacturer's instructions. Finally, DNA was eluted in $200 \mu \mathrm{L}$ of elution buffer and the extract was stored at $-20{ }^{\circ} \mathrm{C}$ until used in PCR amplifications.

\section{Nested PCR amplification}

Enterocytozoon bieneusi was identified via nested-PCR amplification of a $390 \mathrm{bp}$ region of the internal transcribed spacer (ITS) of nuclear ribosomal DNA for each sample [4]. The nestedPCR primers EBITS3: 5' - GGTCATAGGGATGAAGAG - $3^{\prime}$ and EBITS4: 5' - TTCGAGTTCTTTCGCG- CTC - 3' were used for the external reaction, and EBITS1: $5^{\prime}$ - GCTCTGAATATCTATGGCT $-3^{\prime}$ and EBITS2.4: $5^{\prime}$ - ATCGCCGACGGATCCAAGTG $-3^{\prime}$ for the internal reaction. PCR cycling conditions were an external cycle of $94{ }^{\circ} \mathrm{C}$ for $5 \mathrm{~min}$, followed by 35 cycles of $94{ }^{\circ} \mathrm{C}$ for $30 \mathrm{~s}, 57{ }^{\circ} \mathrm{C}$ for $30 \mathrm{~s}\left(55^{\circ} \mathrm{C}\right.$ for the internal reaction), and $72{ }^{\circ} \mathrm{C}$ for $40 \mathrm{~s}$, and a final extension of $72{ }^{\circ} \mathrm{C}$ for 8 min. $2 \times$ EasyTaq PCR SuperMix (TransGene Biotech Co., Beijing, China) were used for each PCR amplification. To ensure accuracy and rule out contamination, DNA from dog-derived genotype D was used as the positive control, and distilled water devoid of DNA as the negative control in each PCR.

\section{Sequencing phylogenetic analyses}

Positive secondary PCR amplicons were sequenced by a commercial sequencing company (GENEWIZ, Suzhou, China). The sequence accuracy was confirmed via bidirectional sequencing, and the sequences obtained were aligned using ClustalX 2.1 (http://www.clustal.org/) with reference sequences downloaded from GenBank (https://www.ncbi.nlm.nih.gov/ genbank/) to determine the species and genotypes. Only the ITS region should be considered when designating the new E. bieneusi genotypes [13]. Representative sequences of the isolated genotypes were submitted to GenBank at the National Center for Biotechnology Information under the following accession numbers: MW412816-MW412818.

Bayesian inference (BI) and Monte Carlo Markov chain methods were used to construct the phylogenetic trees in 
Table 1. Distributions of E. bieneusi in pet dogs from Xinjiang, China.

\begin{tabular}{|c|c|c|c|c|c|}
\hline Category & $\begin{array}{c}\text { No. } \\
\text { sampled }\end{array}$ & $\begin{array}{c}\text { No. } \\
\text { Positive }\end{array}$ & $\begin{array}{l}\text { Infection } \\
{[95 \% \mathrm{CI}]}\end{array}$ & $\begin{array}{l}\chi^{2} \text {-value, } \\
p \text {-value }\end{array}$ & $\begin{array}{l}\text { Enterocytozoon bieneusi } \\
\text { genotypes (no.) }\end{array}$ \\
\hline \multicolumn{6}{|l|}{ Collection site } \\
\hline Urumqi & 191 & 18 & $9.4 \%[5.2-13.6]$ & & EbpC (9), PtEb IX (5), D (2), CD9 (1), CD11 (1) \\
\hline Korla & 137 & 5 & $3.6 \%[0.5-6.8]$ & $4.080,0.043$ & PtEb IX (2), EbpC (2), CD12 (1) \\
\hline Hotan & 213 & 7 & $3.3 \%[0.9-5.7]$ & $6.535,0.011$ & PtEb IX (5), Type IV (1), CD13 (1) \\
\hline Aksu & 22 & 3 & $13.6 \%[0-29.2]$ & $0.394,0.530$ & PtEb IX (2), EbpC (1) \\
\hline Shihezi & 41 & 5 & $12.2 \%[1.7-22.7]$ & $0.290,0.590$ & PtEb IX (5) \\
\hline \multicolumn{6}{|l|}{ Sample source } \\
\hline Pet hospital & 134 & 9 & $6.7 \%[2.4-11.0]$ & & $\operatorname{EbpC}(5), \mathrm{D}(2), \mathrm{PtEb} \mathrm{IX}(1), \mathrm{CD} 11$ (1) \\
\hline Pet shop & 256 & 16 & $6.3 \%[3.3-9.2]$ & $0.032,0.858$ & PtEb IX (7), EbpC (5), CD9 (1), Type IV (1), CD12 (1), CD13 (1) \\
\hline Pet kennel & 214 & 13 & $6.1 \%[2.8-9.3]$ & $0.057,0.811$ & PtEb IX (11), EbpC (2) \\
\hline \multicolumn{6}{|r|}{ - } \\
\hline Female & 326 & 24 & $7.4 \%[4.5-10.2]$ & & $\begin{array}{l}\text { PtEb IX (12), EbpC (6), D (2), CD9 (1), } \\
\text { Type IV (1), CD11 (1), CD12 (1) }\end{array}$ \\
\hline Male & 278 & 14 & $5.0 \%[2.4-7.6]$ & $1.377,0.241$ & PtEb IX (7), EbpC (6), CD13 (1) \\
\hline \multicolumn{6}{|r|}{${ }^{2}$} \\
\hline$\leq 1$ year & 459 & 23 & $5.0 \%[3.0-7.0]$ & & $\begin{array}{l}\text { PtEb IX (8), EbpC (7), D (2), CD9 (1), Type IV (1), } \\
\text { CD11 (1), New2 (1), CD13 (1) }\end{array}$ \\
\hline$>1$ year & 145 & 15 & $10.3 \%[5.3-15.4]$ & $5.318,0.021$ & PtEb IX (10), EbpC (5) \\
\hline Total & 604 & 38 & $6.3 \%[4.3-8.2]$ & & $\begin{array}{l}\text { PtEb IX (19), EbpC (12), D (2), CD9 (1), Type IV (1), CD11 (1), } \\
\text { CD12 (1), CD13 (1) }\end{array}$ \\
\hline
\end{tabular}

MrBayes (version 3.2.6) (http://nbisweden.github.io/MrBayes/). The posterior probability values were calculated by running $1,000,000$ generations. A $50 \%$ majority-rule consensus tree was constructed from the final $75 \%$ of the trees generated via BI. Analyses were run 3 times to ensure convergence and insensitivity to priors.

\section{Statistical analysis}

All statistical analyses were performed using SPSS 22.0 software. In the univariate analyses, a Fisher's exact test was used to compare the prevalence of the E. bieneusi infections in groups constructed according to collection sites, sample source, sex, and age. The infection rates between groups of various sources were compared using a chi-square test. Significant differences were accepted when the $p$-value was $<0.05$.

\section{Results}

\section{Prevalence of $E$. bieneusi in pet dogs}

Among the collected 604 fecal samples collected from pet dogs, 6.3\% (38/604) were positive for E. bieneusi. Specifically, a higher prevalence of $E$. bieneusi infections was observed in Aksu (13.6\%, 2/22), Shihezi (12.2\%, 5/41), and Urumqi (9.9\%, $18 / 191)$. Lower prevalence was observed in Korla (3.6\%, 5/137) and Hotan $(3.3 \%, 7 / 213)$. Among the collection sites, the prevalences of E. bieneusi infections from pet dogs in Korla $\left(\chi^{2}=4.080, p=0.043\right)$ and Hotan $\left(\chi^{2}=6.535, p=0.011\right)$ were statistically lower than the other cities investigated (Table 1).

\section{Enterocytozoon bieneusi infections by sample source, sex, and age group}

Different rates of E. bieneusi infections in pet dogs were observed in different groups (sample source, sex, and age) in the present study. For the different sample sources, 3 sample sources were involved. The prevalences of $E$. bieneusi infections in pet hospitals, pet shops, and pet kennels were $6.7 \%(9 / 134)$, $6.3 \%$ (16/256), and 6.1\% (13/214), respectively. There were no statistically significant differences observed among the different collection sites $\left(\chi^{2}=0.057, p=0.811\right)$. For sex, although the female animals $(7.4 \%, 24 / 326)$ were slightly higher than males $(5.0 \%, 14 / 278)$, the differences were not statistically significant $\left(\chi^{2}=1.377, p=0.241\right)$. For age groups, adult $(>1$ year $)$ dogs $(10.3 \%, 15 / 145)$ were infected at a higher rate than were juvenile $(\leq 1$ year $) \operatorname{dogs}(5.0 \%, 23 / 459)\left(\chi^{2}=5.318, p=0.021\right.$, Table 1).

\section{Genotype distributions and sequence analysis of $E$. bieneusi}

The sequence analysis of 38 positive samples revealed the presence of eight different genotypes in pet dogs in Xinjiang, China. Five of them (PtEb IX, EbpC, D, CD9, and Type IV) were known genotypes and 3 (CD11, CD12, CD13) were novel genotypes. The most prevalent genotype was PtEb IX, observed in $50.0 \%(19 / 38)$ of samples, followed by $\mathrm{EbpC}(31.6 \%$, $12 / 38)$, and D $(5.3 \%, 2 / 38)$. The remaining genotypes (CD9, Type IV, CD11, CD12, CD13) were each observed in one $(2.6 \%, 1 / 38)$ sample (Table 1). No mixed infections of E. bieneusi genotypes were identified in the present study.

Genotype PtEb IX was identical to an isolate from dogs (KJ668719) in China. Genotype EbpC was identical to an isolate from dogs (MN902235) in China. Genotype D was identical to a human isolate (MN136771) from China. Genotype Type IV was identical to a Chinese isolate from a hedgehog (MK841506).

The novel genotypes CD11 (D559) and CD12 (D756) had $99.74 \%$ and $99.23 \%$ homology to the previously isolate identified in a wild boar (MK681466), respectively. These 
Table 2. Prevalence and genotype distributions of E. bieneusi in dogs.

\begin{tabular}{|c|c|c|c|c|}
\hline Collection site & Sample source & $\begin{array}{c}\text { Infection } \\
\text { (no. positive/sampled) }\end{array}$ & Genotype distributions (no.) & References \\
\hline Australia (Victoria) & Domestic dog & $4.4 \%(15 / 342)$ & PtEb IX (9), D (5), VIC_dog1 (1) & [32] \\
\hline China (Heilongjiang) & Pet and stray dog & $6.7 \%(18 / 267)$ & $\begin{array}{l}\text { PtEb IX (12), EbpC (1), D (1), NED2 (1), EbpC/NED1 (1), } \\
\text { PtEb IX/NED3 (1), PtEb IX/NED4 (1) }\end{array}$ & [14] \\
\hline China (Changchun) & Pet market dogs & $7.7 \%(2 / 26)$ & CHN5 (1), CHN6 (1) & [31] \\
\hline China (Shanghai) & $\begin{array}{l}\text { Household, pet } \\
\text { shops, veterinary } \\
\text { clinic dogs }\end{array}$ & $6.0 \%(29 / 485)$ & PtEb IX (28), D (1) & [30] \\
\hline China (Guangzhou) & Pets dogs & $22.9 \%(149 / 651)$ & $\begin{array}{l}\text { PtEb IX (56), GD1 (38), GD2 (24), D (12), EbpC (7), } \\
\text { CD9 (4), I (2), GD3 (2), GD4 (2), GD5 (1), GD6 (1) }\end{array}$ & {$[28]$} \\
\hline $\begin{array}{l}\text { China (Anhui, } \\
\text { Zhejiang) }\end{array}$ & $\begin{array}{l}\text { Veterinary hospitals } \\
\text { dogs }\end{array}$ & $8.6 \%(27 / 315)$ & PtEb IX(16), EbpC(4), CHD 3(3), CHD1(2), CHD2 (2) & [15] \\
\hline $\begin{array}{l}\text { China (Henan, } \\
\text { Sichuan, Shaanxi, } \\
\text { Chongqing) }\end{array}$ & Pet and stray dogs & $15.5 \%(54 / 348)$ & $\begin{array}{l}\text { PtEb IX (26), CD8 (4), O (4), D (3), CD7 (2), EbpA (2), } \\
\text { CM1 (2), EbpC (1), Peru8 (1), type IV (1), } \\
\text { PigEBITS5 (1), CD1 (1), CD2 (1), CD3 (1), CD4 (1), } \\
\text { CD5 (1), CD6 (1), CD9 (1) }\end{array}$ & [11] \\
\hline Colombia (Bogota) & Stray dogs & $15.0 \%(18 / 120)$ & PtEb IX (16), type IV (1), Peru 5/WL11 (1) & [25] \\
\hline Egypt (Giza) & Domestic dogs & $13.0 \%(14 / 108)$ & N/A & [2] \\
\hline Iran (Tehran) & Stray dogs & $5.3 \%(4 / 75)$ & D (4) & [7] \\
\hline Iran (Ardebil) & Ownership dogs & $11.8 \%(2 / 17)$ & N/A & {$[3]$} \\
\hline Iran (Isfahan) & Animal clinic dogs & $25.8 \%(8 / 100)$ & N/A & {$[10]$} \\
\hline $\begin{array}{l}\text { Japan (Osaka, } \\
\text { Ishikawa, Niigata) }\end{array}$ & Pet and stray dogs & $2.5 \%(2 / 79)$ & PtEb IX (2) & {$[1]$} \\
\hline Japan & Family pet dogs & $4.4 \%(26 / 597)$ & PtEb IX (26) & {$[21]$} \\
\hline Poland & Household dogs & $4.9 \%(4 / 82)$ & $\mathrm{PtEb}$ IX (2), D (2) & {$[22]$} \\
\hline Portugal (Lisbon) & $\begin{array}{r}\text { Pet owners and } \\
\text { shelters dogs }\end{array}$ & $3^{\mathrm{a}}$ & PtEb IX (1), Peru 6 (1); D/Peru 9 (1) & [17] \\
\hline Spain (Galicia) & Domestic dogs & $11.8 \%(2 / 17)$ & N/A & [18] \\
\hline Spain & Domestic dogs & $9.6 \%(7 / 73)$ & A (7) & [9] \\
\hline Spain (Madrid) & Domestic dogs & $8.7 \%(4 / 46)$ & N/A & [6] \\
\hline Spain (Álava) & $\begin{array}{l}\text { Owned and sheltered } \\
\text { dogs }\end{array}$ & $0.8 \%(2 / 237)$ & PtEb IX (1), BEB6 (1) & [5] \\
\hline Switzerland & Farm dogs & $8.3 \%(3 / 36)$ & PtEb IX (3) & [19] \\
\hline
\end{tabular}

N/A, not available; Bold $=$ dominant genotype.

${ }^{a}$ This article only reports the genetic diversity of samples previously identified as E. bieneusi-positive.

two isolates had 1 and 3 substitutions to isolate MK681466, at $110(\mathrm{~T} \rightarrow \mathrm{C})$, and $106(\mathrm{~T} \rightarrow \mathrm{C}), 141(\mathrm{~A} \rightarrow \mathrm{G})$, and 169 $(\mathrm{T} \rightarrow \mathrm{C})$, respectively. The novel genotype CD13 (D1338) had $99.74 \%$ homology to fox isolate MN029060 in China, with one substitution at $162(\mathrm{~T} \rightarrow \mathrm{C})$.

\section{Phylogenetic analyses}

Bayesian inference phylogenetic analysis revealed that the 3 known (EbpC, D, and Type IV) genotypes and 3 novel (CD11, CD12, CD13) genotypes identified herein clustered into Group 1, which suggests zoonotic potential. The other genotypes PtEb IX and CD9 were previously assigned to Group 11, which tends to exhibit host specificity (Fig. 1).

\section{Discussion}

In the present study, a $6.3 \%(38 / 604)$ E. bieneusi positivity rate among pet dogs in Xinjiang, China was observed. A significantly higher infection rate was observed in pet dogs $(22.9 \%$, 149/651) in Guangdong, China [28], and pet and stray dogs
$(15.5 \%, 54 / 348)$ in China in another study [11], domestic dogs $(11.7 \%, 2 / 17 ; 9.6 \%, 7 / 73)$ in 2 studies in Spain [9, 18], and stray dogs $(15.0 \%, 18 / 120)$ in Colombia [25]. Significantly lower infection rates were reported in owned and sheltered dogs $(0.8 \%, 2 / 237)$ in Northern Spain [5], family pet dogs $(4.4 \%$, $26 / 597)$ in Japan [21], and domestic dogs $(4.4 \%, 15 / 342)$ in Australia [32]. However, similar results were reported in pet dogs $(6.0 \%, 29 / 485)$ in Shanghai, China [30], and in pet and stray dogs $(6.7 \%, 18 / 267)$ in Heilongjiang, China [14].

Among the facility types (pet hospital, pet shop, and pet kennel) and sexes (female and male) from which fecal samples were collected in the present study, no differences in E. bieneusi positivity were observed (Table 1). For the age groups, the prevalence of $E$. bieneusi infections in adult ( $>1$ year) dogs $(10.3 \%, 15 / 145)$ was higher than that in juveniles $(\leq 1$ year, $5.0 \%, 23 / 459)$. This observation was consistent with previous reports in pet and stray dogs (10.1\% vs. 1.8\%) in Heilongjiang, China [14], pet and stray dogs $(12.8 \%$ vs. $6.1 \%)$ throughout China [11], and stray dogs $(18.8 \%$ vs. 0) in Colombia [25]. However, the prevalence cannot be compared due to the differences in study population composition of age, region, and living conditions between the previous and present studies. 


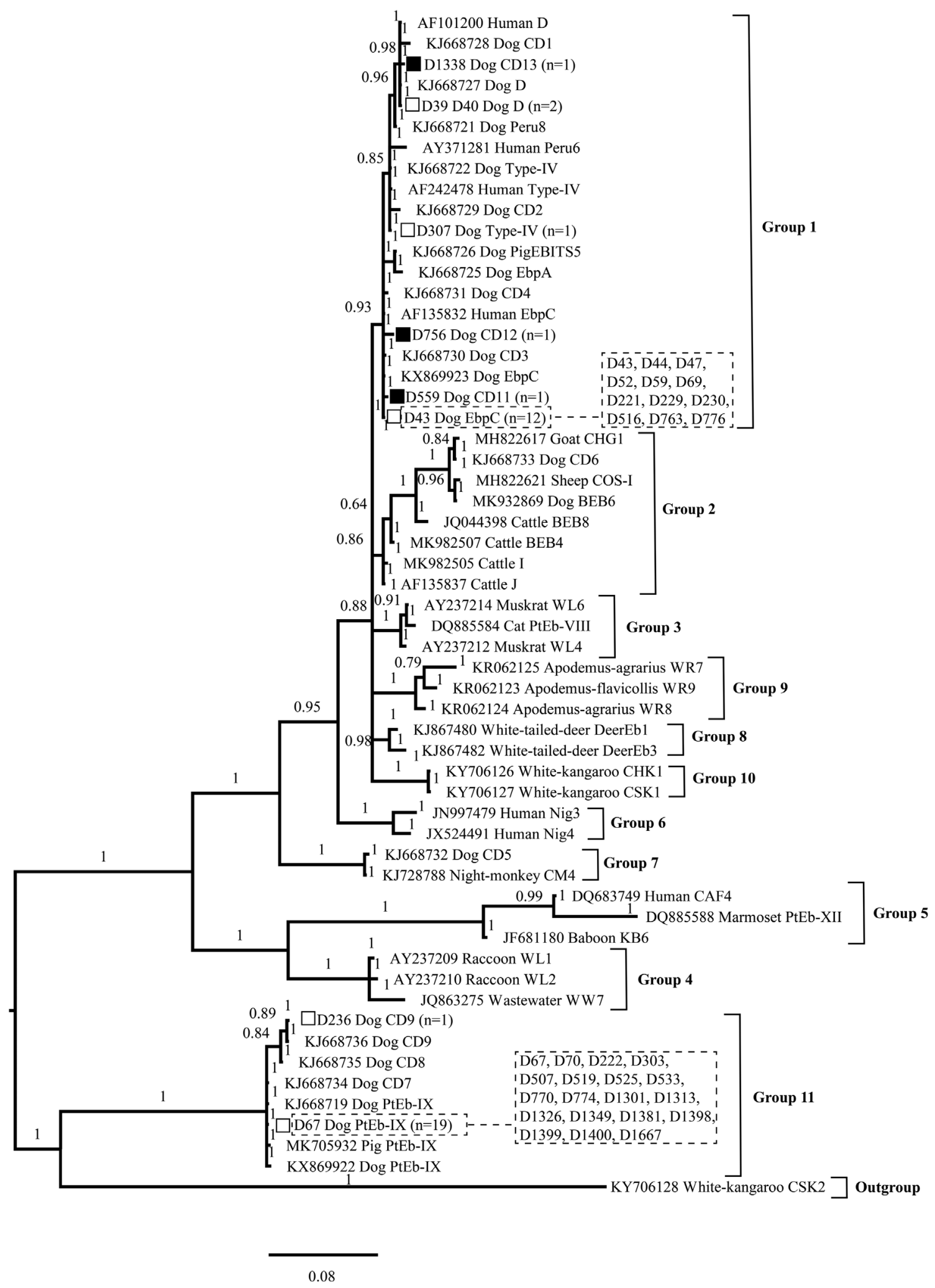

Figure 1. Phylogenetic tree based on Bayesian inference (BI) analysis of the Enterocytozoon bieneusi ITS sequences. Statistically significant posterior probabilities $(>0.7)$ are indicated on the branches. Known and novel E. bieneusi ITS genotypes identified in the present study are indicated by empty and filled squares, respectively. 
In general, the prevalence reported in these studies may be attributable to differences in the geographic area, feeding sites, life-style, age distribution, sample sizes, animals health status, management systems, and population densities of the animals tested, as well as other unidentified factors.

Eight different $E$. bieneusi ITS genotypes were identified in 38 positive pet dogs in the present study. Genotype $\mathrm{PtEb}$ IX was dominant and the most prevalent genotype identified in pet dogs in the present study and in many previous investigations (Table 2). Genotype PtEb IX was primarily isolated from dogs worldwide, and cats in China and Australia, and a wild badger in Spain [11, 24, 32]. Genotype PtEb IX is considered to be a canine host-adapted genotype [16, 20]. Genotypes EbpC and D were identified in 12 and 2 samples, respectively. Both genotypes have been widely identified in humans, non-human primates, and pigs, with occasional reports in dogs, horses, and wildlife in China [29]. Genotype Type IV, identified in one dog sample here, has previously been reported in humans, non-human primates, and wild animals in China [29]. Taken together, the data suggest that these three genotypes have the potential for zoonotic transmission. Since E. bieneusi was mainly transmitted by the fecal-oral route, the assessment of possible sources had been focused on exposure of pet dogs to contaminated soil or water while taking part in outdoor activities. This was partly confirmed in previous studies [5, 22, 28, 30]. Genotype CD9 has previously been identified in one female pet dog sample in Xi' an, Shaanxi Province, China [11]. The remaining 3 novel genotypes (CD11, CD12, CD13), as well as 3 known (EbpC, D, and Type IV) genotypes identified herein clustered into the zoonotic potential Group 1, based on the BI phylogenetic analysis. The other genotypes, PtEb IX and CD9, clustered into Group 11, which indicates some host specificity (Fig. 1). There are several reports of E. bieneusi in humans, farm animals, and wild animals in Xinjiang, China (Supplemental Material Table S1). Identification of the same E. bieneusi genotypes between humans and animals, and different types of animals, indicates the potential likelihood that these genotypes are mutual transmitted between these hosts.

\section{Conclusions}

The results presented here show that the prevalence of E. bieneusi in pet dogs in Xinjiang was relatively low. Present and previous studies indicated that genotypes PtEb IX and CD9 were considered host-adapted genotypes and are unlikely to exhibit zoonotic transmission. Genotypes EbpC, D, and Type IV, and 3 novel genotypes (CD11, CD12, and CD13) possibly exhibit zoonotic transmission potential. To determine the actual threat that these genotypes pose to public health requires further investigation.

\section{Supplemental material}

Supplementary material is available at https://www.parasitejournal.org/10.1051/parasite/2021057/olm

Table S1. Previous reports of Enterocytozoon bieneusi in humans, farm animals, and wild animals in Xinjiang, China.

\section{Conflict of interest}

The authors declare that they have no conflict of interest.

Acknowledgements. This study was supported by the Program for Young and Middle-aged Leading Science, Technology, and Innovation of the Xinjiang Production \& Construction Corps (2018CB034), and the National College Students' Innovation and Entrepreneurship Training Program (201910757026).

\section{References}

1. Abe N, Kimata I, Iseki M. 2009. Molecular evidence off Enterocytozoon bieneusi in Japan. Journal of Veterinary Medical Science, 71(2), 217-219.

2. Al-Herrawy AZ, Gad MA. 2016. Microsporidial spores in fecal samples of some domesticated animals living in Giza, Egypt. Iranian Journal of Parasitology, 11(2), 195-203.

3. Askari Z, Mirjalali H, Mohebali M, Zarei Z, Shojaei S, Rezaeian T, Rezaeian M. 2015. Molecular detection and identification of zoonotic microsporidia spore in fecal samples of some animals with close-contact to human. Iranian Journal of Parasitology, 10(3), 381-388.

4. Buckholt MA, Lee JH, Tzipori S. 2002. Prevalence of Enterocytozoon bieneusi in swine: an 18-month survey at a slaughterhouse in Massachusetts. Applied and Environmental Microbiology, 68(5), 2595-2599.

5. Dashti A, Santín M, Cano L, de Lucio A, Bailo B, de Mingo MH, Köster PC, Fernández-Basterra JA, Aramburu-Aguirre J, López-Molina N, Fernández-Crespo JC, Calero-Bernal R, Carmena D. 2019. Occurrence and genetic diversity of Enterocytozoon bieneusi (Microsporidia) in owned and sheltered dogs and cats in Northern Spain. Parasitology Research, 118(10), 2979-2987.

6. del Águila C, Izquierdo F, Navajas R, Pieniazek NJ, Miró G, Alonso AI, Da Silva AJ, Fenoy S. 1999. Enterocytozoon bieneusi in animals: rabbits and dogs as new hosts. Journal of Eukaryotic Microbiology, 46(5), 8S-9S.

7. Delrobaei M, Jamshidi S, Shayan P, Ebrahimzade E, Ashrafi Tamai I, Rezaeian M, Mirjalali H. 2019. Molecular detection and genotyping of intestinal microsporidia from stray dogs in Iran. Iranian Journal of Parasitology, 14(1), 159-166.

8. Desportes I, Le Charpentier Y, Galian A, Bernard F, CochandPriollet B, Lavergne A, Ravisse P, Modigliani R. 1985. Occurrence of a new microsporidan: Enterocytozoon bieneusi n.g., n. sp., in the enterocytes of a human patient with AIDS. Journal of Protozoology, 32(2), 250-254.

9. Galván-Díaz AL, Magnet A, Fenoy S, Henriques-Gil N, Haro M, Gordo FP, Millán J, Miró G, del Águila C, Izquierdo F. 2014. Microsporidia detection and genotyping study of human pathogenic E. bieneusi in animals from Spain. PLoS One, 9(3), e92289.

10. Jamshidi Sh, Tabrizi AS, Bahrami M, Momtaz H. 2012. Microsporidia in household dogs and cats in Iran; a zoonotic concern. Veterinary Parasitology, 185(2-4), 121-123.

11. Karim MR, Dong H, Yu F, Jian F, Zhang L, Wang R, Zhang S, Rume FI, Ning C, Xiao L. 2014. Genetic diversity in Enterocytozoon bieneusi isolates from dogs and cats in China: host specificity and public health implications. Journal of Clinical Microbiology, 52(9), 3297-3302.

12. Li J, Jiang Y, Wang W, Chao L, Jia Y, Yuan Y, Wang J, Qiu J, Qi M. 2020. Molecular identification and genotyping of Enterocytozoon bieneusi in experimental rats in China. Experimental Parasitology, 210, 107850. 
13. Li W, Feng Y, Xiao L. 2020. Diagnosis and molecular typing of Enterocytozoon bieneusi: the significant role of domestic animals in transmission of human microsporidiosis. Research in Veterinary Science, 133, 251-261.

14. Li W, Li Y, Song M, Lu Y, Yang J, Tao W, Jiang Y, Wan Q, Zhang S, Xiao L. 2015. Prevalence and genetic characteristics of Cryptosporidium, Enterocytozoon bieneusi and Giardia duodenalis in cats and dogs in Heilongjiang province, China. Veterinary Parasitology, 208(3-4), 125-134.

15. Li WC, Qin J, Wang K, Gu YF. 2018. Genotypes of Enterocytozoon bieneusi in dogs and cats in Eastern China. Iranian Journal of Parasitology, 13(3), 457-465.

16. Li W, Feng Y, Santin M. 2019. Host specifcity of Enterocytozoon bieneusi and public health implications. Trends in Parasitology, 35, 436-451.

17. Lobo ML, Xiao L, Cama V, Stevens T, Antunes F, Matos O. 2006. Genotypes of Enterocytozoon bieneusi in mammals in Portugal. Journal of Eukaryotic Microbiology, 53(Suppl 1), S61-64.

18. Lores B, del Aguila C, Arias C. 2002. Enterocytozoon bieneusi (microsporidia) in faecal samples from domestic animals from Galicia. Spain. Memórias do Instituto Oswaldo Cruz, 97(7), 941-945.

19. Mathis A, Breitenmoser AC, Deplazes P. 1999. Detection of new Enterocytozoon genotypes in faecal samples of farm dogs and a cat. Parasite, 6(2), 189-193.

20. Ou Y, Jiang W, Roellig DM, Wan Z, Li N, Guo Y, Feng Y, Xiao L. 2020. Characterizations of Enterocytozoon bieneusi at new genetic loci reveal a lack of strict host specificity among common genotypes and the existence of a canine-adapted Enterocytozoon species. International Journal for Parasitology, 51(2-3), 215-223.

21. Phrompraphai T, Itoh N, Iijima Y, Ito Y, Kimura Y. 2019. Molecular detection and genotyping of Enterocytozoon bieneusi in family pet dogs obtained from different routes in Japan. Parasitology International, 70, 86-88.

22. Piekarska J, Kicia M, Wesoõõlowska M, Kopacz Ż, Gorczykowski M, Szczepankiewicz B, Kváč M, Sak B. 2017. Zoonotic microsporidia in dogs and cats in Poland. Veterinary Parasitology, 246, 108-111.
23. Sak B, Brady D, Pelikánová M, Květoňová D, Rost M, Kostka M, Tolarová V, Hůzová Z, Kváč M. 2011. Unapparent microsporidial infection among immunocompetent humans in the Czech Republic. Journal of Clinical Microbiology, 49(3), 1064-1070.

24. Santín M, Calero-Bernal R, Carmena D, Mateo M, Balseiro A, Barral M, Lima Barbero JF, Habela MÁ. 2018. Molecular characterization of Enterocytozoon bieneusi in wild carnivores in Spain. Journal of Eukaryotic Microbiology, 65(4), 468-474.

25. Santín M, Cortés Vecino JA, Fayer R. 2008. Enterocytozoon bieneusi genotypes in dogs in Bogota, Colombia. American Journal of Tropical Medicine and Hygiene, 79(2), 215-217.

26. Stark D, van Hal S, Barratt J, Ellis J, Marriott D, Harkness J. 2009. Limited genetic diversity among genotypes of Enterocytozoon bieneusi strains isolated from HIV-infected patients from Sydney, Australia. Journal of Medical Microbiology, 58(3), 355-357.

27. Stentiford GD, Becnel -J, Weiss LM, Keeling PJ, Didier ES, Williams BP, Bjornson S, Kent ML, Freeman MA, Brown MJF, Troemel ER, Roesel K, Sokolova Y, Snowden KF, Solter L. 2016. Microsporidia - emergent pathogens in the global food chain. Trends in Parasitology, 32, 336-348.

28. Wang H, Lin X, Sun Y, Qi N, Lv M, Xiao W, Chen Y, Xiang R, Sun M, Zhang L. 2020. Occurrence, risk factors and genotypes of Enterocytozoon bieneusi in dogs and cats in Guangzhou, southern China: high genotype diversity and zoonotic concern. BMC Veterinary Research, 16(1), 201.

29. Wang SS, Wang RJ, Fan XC, Liu TL, Zhang LX, Zhao GH. 2018. Prevalence and genotypes of Enterocytozoon bieneusi in China. Acta Tropica, 183, 142-152.

30. Xu H, Jin Y, Wu W, Li P, Wang L, Li N, Feng Y, Xiao L. 2016. Genotypes of Cryptosporidium spp., Enterocytozoon bieneusi and Giardia duodenalis in dogs and cats in Shanghai, China. Parasites \& Vectors, 9, 121.

31. Zhang X, Wang Z, Su Y, Liang X, Sun X, Peng S, Lu H, Jiang N, Yin J, Xiang M, Chen Q. 2011. Identification and genotyping of Enterocytozoon bieneusi in China. Journal of Clinical Microbiology, 49(5), 2006-2008.

32. Zhang Y, Koehler AV, Wang T, Cunliffe D, Gasser RB. 2019. Enterocytozoon bieneusi genotypes in cats and dogs in Victoria, Australia. BMC Microbiology, 19(1), 183.

Cite this article as: Cao Y, Tong Q, Zhao C, Maimaiti A, Chuai L, Wang J, Ma D \& Qi M. 2021. Molecular detection and genotyping of Enterocytozoon bieneusi in pet dogs in Xinjiang, Northwestern China. Parasite 28, 57.

\section{PARASTE}

An international open-access, peer-reviewed, online journal publishing high quality papers on all aspects of human and animal parasitology

Reviews, articles and short notes may be submitted. Fields include, but are not limited to: general, medical and veterinary parasitology; morphology, including ultrastructure; parasite systematics, including entomology, acarology, helminthology and protistology, and molecular analyses; molecular biology and biochemistry; immunology of parasitic diseases; host-parasite relationships; ecology and life history of parasites; epidemiology; therapeutics; new diagnostic tools.

All papers in Parasite are published in English. Manuscripts should have a broad interest and must not have been published or submitted elsewhere. No limit is imposed on the length of manuscripts.

Parasite (open-access) continues Parasite (print and online editions, 1994-2012) and Annales de Parasitologie Humaine et Comparée (1923-1993) and is the official journal of the Société Française de Parasitologie. 